THERAPEUTICS

\section{Brief encounter}

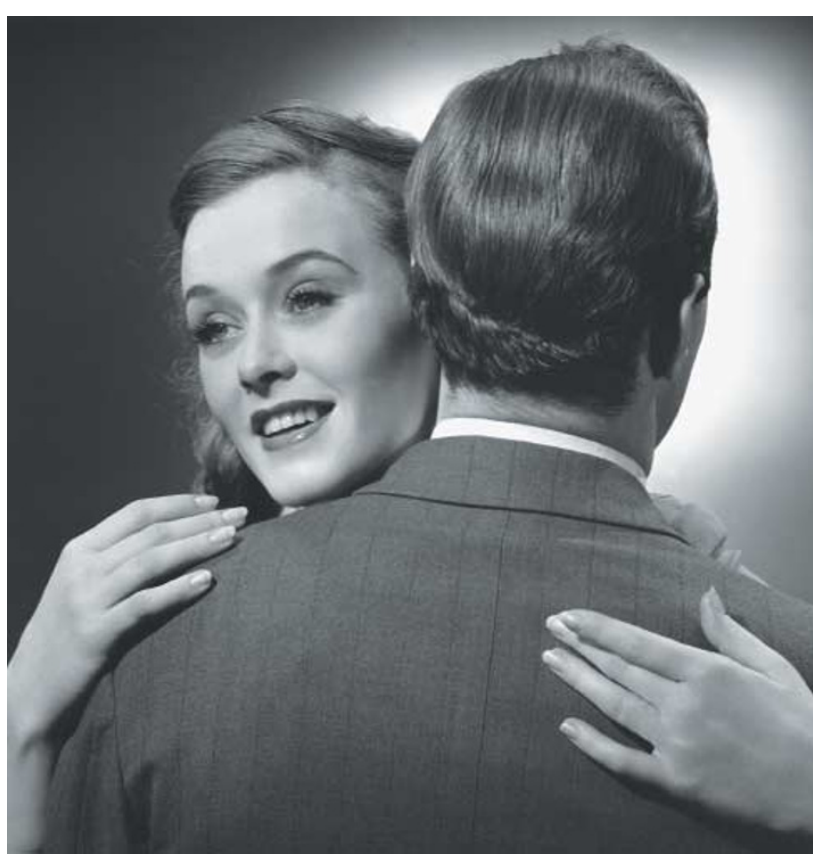

Both preclinical and clinical trials show that anticancer therapies that disrupt the blood supply of a solid tumour are most effective when combined with either radio- or chemotherapy. Jain and colleagues have now found a possible explanation for this result and, surprisingly, it is not that anti-angiogenic factors eradicate the tumour's vasculature, but that they might transiently improve it.

The vascular network feeding a solid tumour is often a mass of highly disorganized and overly large, leaky vessels, leading to an inefficient blood supply and mostly hypoxic tumour tissue. The treatment of solid tumours, such as glioblastoma multiforme, with radiotherapy is hampered by these hypoxic conditions. Jain and colleagues used a mouse orthotopic model of human glioblastoma to assess why combined anti-angiogenic treatment and radiotherapy gives better results.

Initially, the authors combined an antibody (DC101) that inhibits vascular endothelial growth factor receptor 2 (VEGFR2) with $\gamma$-radiation and examined tumour growth. Although both treatments together showed an additive response in delaying tumour growth, scheduling radiation treatment 4-6 days after starting treatment with DC101 gave a synergistic response. However, delaying radiotherapy until 8 days after DC101 treatment resulted in loss of this synergy. Jain and co-workers thought that this might be due to a transient improvement in the vascular network, increasing the levels of tumour oxygenation and making the tumour cells more sensitive to radiation.

Analysis of the tumours using in vivo multiphoton microscopy and immunofluoresence indicated that the tumour vasculature did change during the initial stages of treatment with DC101 — the vessels became less tortuous and vessel diameter decreased. These changes correlated with the recruitment of pericytes to the tumour vessels - cells that are associated with stabilized vessels in normal tissues. cDNA microarray data, along with mRNA and protein studies showed that angiopoietin-1
VIRAL CARCINOGENESIS

\section{Reactivation}

Patients with rheumatoid arthritis and polymyositis treated with the immunosuppresive drug methotrexate (MTX) develop Epstein-Barr virus (EBV)positive lymphomas more frequently than healthy individuals or similar patients treated with non-immunosuppressive drugs. Wen-hai Feng, Jeffrey I. Cohen et al. now report that MTX might promote EBVpositive lymphomas in these patients by a combination of immunosuppression and reactivation of latent $\mathrm{EBV}$.

Latent infection is associated with cellular transformation of B cells, but the lytic form of infection, which leads to release of virus particles and death of the host cell, might also increase the number of latently EBV-infected $B$ cells and increase the likelihood of malignancy. Using pharmacological doses of MTX, the authors showed that MTX induced lytic infection in an EBV-positive latently infected gastric carcinoma cell line (AGS-EBV-GFP) and in EBV-positive lymphoblastoid cell lines (LCLs). Other drugs used to treat rheumatoid arthritis did not have this effect.

To investigate whether MTX induced lytic EBV gene expression directly Feng and Cohen et al. transfected EBV-negative cells with the promoters of the early lytic viral proteins BZLF1 and BRLF1 linked to a reporter gene and treated them with MTX the expression of the reporter gene more than doubled in both experiments. But is this expression accompanied by replication of lytic EBV DNA? Treatment of AGS-EBV-GFP cells with MTX increased the copies of the lytic EBV genome and this effect was inhibited by addition of the antiviral drug acyclovir. This induction of replication seems to be MTX specific, because gemcitabine, which also induced lytic viral gene expression, did not increase replication of EBV DNA. Furthermore, when drug-free medium was taken from MTX-treated AGS-EBV-GFP cells and used to infect an EBV-positive Burkitt's lymphoma cell line, GFP expression was detected in the resulting infected cells, indicating that MTX had induced release of infectious virions.

So what effect does MTX have on EBV loads in patients with rheumatoid arthritis or polymyositis? The mean EBV load in 29 patients receiving treatment regimens including MTX was $40 \mathrm{EBV}$ copies per $10^{6}$ cellular genomes, compared with $5.1 \mathrm{EBV}$ copies in the 12 patients on regimens that did not include MTX.

The authors conclude that the unique ability of MTX to induce EBV replication at the same time as suppressing the immune system might explain the association with EBV-positive lymphomas in patients who already have an increased risk of these malignancies. Whether MTX treatment of cancer patients has any effect on EBV reactivation or any link with EBV-associated tumours should be investigated.

\section{Ezzie Hutchinson}

(2) References and links ORIGINAL RESEARCH PAPER Feng, W. et al. Reactivation of latent Epstein-Barr virus by methotrexate: a potential contributor to methotrexate-associated lymphomas. J. Natl Cancer Inst. 96, 1691-1702 (2004) FURTHER READING Young, L. S. \& Rickinson, A. B. Epstein-Barr virus: 40 years on. Nature Rev. Cancer 4, 757-768 (2004)

Shannon Kenney's lab:

http://cancer.med.unc.edu/research/faculty/displayMember. asp?ID $=100$ 
